\title{
Hom-Lie Triple System and Hom-Bol Algebra Structures on Hom-Maltsev and Right Hom-Alternative Algebras
}

\author{
Sylvain Attan and A. Nourou Issa \\ Département de Mathématiques, Université d'Abomey-Calavi, 01 BP 4521 Cotonou, Benin \\ Correspondence should be addressed to A. Nourou Issa; woraniss@yahoo.fr
}

Received 18 December 2017; Accepted 6 March 2018; Published 2 May 2018

Academic Editor: Kaiming Zhao

Copyright (C) 2018 Sylvain Attan and A. Nourou Issa. This is an open access article distributed under the Creative Commons Attribution License, which permits unrestricted use, distribution, and reproduction in any medium, provided the original work is properly cited.

\begin{abstract}
Every multiplicative Hom-Maltsev algebra has a natural multiplicative Hom-Lie triple system structure. Moreover, there is a natural Hom-Bol algebra structure on every multiplicative Hom-Maltsev algebra and on every multiplicative right (or left) Hom-alternative algebra.
\end{abstract}

\section{Introduction}

The study of Lie triple systems (Lts) on their own as algebraic objects started from Jacobson's work [1] and developed further by, for example, Lister [2], Yamaguti [3], and other mathematicians. Lts constitute examples of ternary algebras. If $(g,[]$,$) is a Lie algebra, then (g,[,]$,$) is a Lts, where$ $[x, y, z]:=[[x, y], z]$ (see $[1,4,5])$. Another construction of Lts from binary algebras is the one from Maltsev algebras found by Loos [6].

Maltsev algebras were introduced by Maltsev [7] in a study of commutator algebras of alternative algebras and also as a study of tangent algebras to local smooth Moufang loops. Maltsev used the name "Moufang-Lie algebras" for these nonassociative algebras while Sagle [8] introduced the term "Malcev algebras." Equivalent defining identities of Maltsev algebras are pointed out in [8].

Alternative algebras, Maltsev algebras, and Lts (among other algebras) received a twisted generalization in the development of the theory of Hom-algebras during these latest years. The forerunner of the theory of Hom-algebras is the Hom-Lie algebra introduced by Hartwig et al. in [9] in order to describe the structure of some deformation of the Witt algebra and the Virasoro algebra. It is well-known that Lie algebras are related to associative algebras via the commutator bracket construction. In the search of a similar construction for Hom-Lie algebras, the notion of a Homassociative algebra is introduced by Makhlouf and Silvestrov in [10], where it is proved that a Hom-associative algebra gives rise to a Hom-Lie algebra via the commutator bracket construction. Since then, various Hom-type structures are considered (see, e.g., [11-23]). Roughly speaking, Homalgebraic structures are corresponding ordinary algebraic structures whose defining identities are twisted by a linear self-map. A general method for constructing a Hom-type algebra from the ordinary type of algebra with a linear selfmap is given by Yau in [24].

In $[11,21], n$-ary Hom-algebra structures generalizing $n$ ary algebras of Lie type or associative type were considered. In particular, generalizations of $n$-ary Nambu or NambuLie algebras, called $n$-ary Hom-Nambu and Hom-NambuLie algebras, respectively, were introduced in [11] while Hom-Jordan algebras were defined in [18] and Hom-Lie triple systems (Hom-Lts) were introduced in [21] (another definition of a Hom-Jordan algebra is given in [20]). It is shown [21] that Hom-Lts are ternary Hom-Nambu algebras with additional properties and that Hom-Lts arise also from Hom-Jordan triple systems or from other Hom-type algebras.

Motivated by the relationships between some classes of binary algebras and some classes of binary-ternary algebras, a study of Hom-type generalization of binary-ternary algebras is initiated in [16] with the definition of Hom-Akivis algebras. 
Further, Hom-Lie-Yamaguti algebras are considered in [14] and Hom-Bol algebras [12] are defined as a twisted generalization of Bol algebras which are introduced and studied in [25-27] as infinitesimal structures tangent to smooth Bol loops (some aspects of the theory of Bol algebras are discussed in [28-30]).

In this paper, we will be concerned with right (or left) Hom-alternative algebras, Hom-Maltsev algebras, Hom-Lts, and Hom-Bol algebras. We extend Loos' construction of Lts from Maltsev algebras ([6], Satz 1) to the Hom-algebra setting (Section 3). Specifically, we prove (Theorem 14) that every multiplicative Hom-Maltsev algebra is naturally a multiplicative Hom-Lts by a suitable definition of the ternary operation. As a tool in the proof of this fact, we point out a kind of compatibility relation between the original binary operation of a given Hom-Maltsev algebra and the ternary operation mentioned above (Lemma 13). Moreover, we obtain that every multiplicative Hom-Maltsev algebra has a natural Hom-Bol algebra structure (Theorem 17). In [31] Mikheev proved that every right alternative algebra has a natural (left) Bol algebra structure. In [29] Hentzel and Peresi proved that not only a right alternative algebra but also a left alternative algebra has left Bol algebra structure. In Section 4 we prove that the Hom-analogue of these results holds. Specifically, every multiplicative right (or left) Hom-alternative algebra is a Hom-Bol algebra (Theorem 23). It could be observed that the methods used in the proof of results in $[6,29,31]$ cannot be reported in the Hom-algebra setting at the present stage of the theory of Hom-algebras. In Section 5 we specify Theorem 23 to recover the construction of left Bol algebras from right alternative algebras (Theorem 26; one observes that, in our proof, we use essentially some fundamental properties of right alternative algebras). In Section 2 we recall some basic definitions and facts about Hom-algebras. We define the Hom-Jordan associator of a given Hom-algebra and point out that every Hom-algebra is a Hom-triple system with respect to the Hom-Jordan associator. This observation is used in the proof of Theorem 23.

All vector spaces and algebras are meant over an algebraically closed ground field $\mathbb{K}$ of characteristic 0 .

\section{Some Basics on Hom-Algebras}

We first recall some relevant definitions about binary and ternary Hom-algebras. In particular, we recall the notion of a Hom-Maltsev algebra as well as some of its equivalent defining identities. Although various types of $n$-ary Homalgebras are introduced and discussed in $[11,21]$, for our purpose, we will consider ternary Hom-algebras (ternary HomNambu algebras and Hom-Lts) and Hom-Bol algebras. For fundamentals on Hom-algebras, one may refer, for example, to $[9-11,13,17,24,32]$. Some aspects of the theory of binary Hom-algebras are considered in [33], while some classes of binary-ternary Hom-algebras are defined and discussed in $[12,14,16]$.

Definition 1. (i) A Hom-algebra is a triple $(A, *, \alpha)$ in which $A$ is a $\mathbb{K}$-vector space, $*: A \times A \rightarrow A$ a bilinear map (the binary operation), and $\alpha: A \rightarrow A$ a linear map (the twisting map).
The Hom-algebra $A$ is said to be multiplicative if $\alpha(x * y)=$ $\alpha(x) * \alpha(y)$ for all $x, y \in A$.

(ii) The Hom-Jacobian in $(A, *, \alpha)$ is the trilinear map $J_{\alpha}$ : $A \times A \times A \rightarrow A$ defined as $J_{\alpha}(x, y, z):=\circlearrowleft_{x, y, z}(x * y) *$ $\alpha(z)$, where $\mho_{x, y, z}$ denotes the sum over cyclic permutation of $x, y, z$.

(iii) The Hom-associator of a Hom-algebra $(A, *, \alpha)$ is the trilinear map as : $A^{\otimes 3} \rightarrow A$ defined as as $(x, y, z)=(x * y) *$ $\alpha(z)-\alpha(x) *(y * z)$. If as $(x, y, z)=0$ for all $x, y, z \in A$, then $(A, *, \alpha)$ is said to be Hom-associative.

Remark 2. If $\alpha=$ id (the identity map), then a Homalgebra $(A, *, \alpha)$ reduces to an ordinary algebra $(A, *)$, the Hom-Jacobian $J_{\alpha}$ is the ordinary Jacobian $J$, and the Homassociator is the usual associator for the algebra $(A, *)$. One observes that, in general, the map $\alpha$ is not always injective nor surjective (see $[13,15]$ for discussions on the subject). So, for example, a given algebra can be twisted into zero algebra and some properties of Hom-algebras may not be valid for corresponding ordinary algebras.

As for ordinary algebras, to each Hom-algebra $\mathscr{A}:=$ $(A, *, \alpha)$ are attached two Hom-algebras: the commutator Hom-algebra $\mathscr{A}^{-}:=(A,[],, \alpha)$, where $[x, y]:=x * y-y * x$ (the commutator of $x$ and $y$ ), and the plus Hom-algebra $\mathscr{A}^{+}:=$ $(A, \circ, \alpha)$, where $x \circ y:=x * y+y * x$ (the Jordan product) for all $x, y \in A$.

For our purpose, we provide the following.

Definition 3. The Hom-Jordan associator of a Hom-algebra $\mathscr{A}:=(A, *, \alpha)$ is the trilinear map as ${ }^{J}: A^{\otimes 3} \rightarrow A$ defined as $\operatorname{as}^{J}(x, y, z)=(x \circ y) \circ \alpha(z)-\alpha(x) \circ(y \circ z)$, where "。" is the Jordan product on $A$.

If $\alpha=\mathrm{id}$, the Hom-Jordan associator reduces to the usual Jordan associator.

Definition 4. (i) A Hom-Lie algebra is a Hom-algebra $(A, *, \alpha)$ such that the binary operation " $*$ " is anticommutative and the Hom-Jacobi identity

$$
J_{\alpha}(x, y, z)=0
$$

holds for all $x, y$, and $z$ in $A$ ([9]).

(ii) A Hom-Maltsev algebra is a Hom-algebra $(A, *, \alpha)$ such that the binary operation “*” is anticommutative and that the Hom-Maltsev identity

$$
J_{\alpha}(\alpha(x), \alpha(y), x * z)=J_{\alpha}(x, y, z) * \alpha^{2}(x)
$$

holds for all $x, y, z$ in $A$ ([20]).

(iii) A Hom-Jordan algebra is a Hom-algebra $(A, *, \alpha)$ such that $(A, *)$ is a commutative algebra and the Hom-Jordan identity

$$
\text { as }(x * x, \alpha(y), \alpha(x))=0
$$

is satisfied for all $x, y$ in $A([20])$.

(iv) A Hom-algebra $(A, *, \alpha)$ is called a right Homalternative algebra if

$$
\text { as }(x, y, y)=0
$$


for all $x, y$ in A. A Hom-algebra $(A, *, \alpha)$ is called a left Homalternative algebra if

$$
\text { as }(x, x, y)=0
$$

for all $x, y$ in $A$. A Hom-algebra $(A, *, \alpha)$ is called a Homalternative algebra if it is both right and left Hom-alternative [18].

Remark 5. When $\alpha=$ id, the Hom-Jacobi identity (1) is the usual Jacobi identity $J(x, y, z)=0$. Likewise, for $\alpha=$ id, the Hom-Maltsev identity (2) reduces to the Maltsev identity $J(x, y, x * z)=J(x, y, z) * x$. Therefore a Lie (resp., Maltsev) algebra $(A, *)$ may be seen as a Hom-Lie (resp., Hom-Maltsev) algebra with the identity map as the twisting map. Also Hom-Maltsev algebras generalize HomLie algebras in the same way that Maltsev algebras generalize Lie algebras. For $\alpha=$ id in the Hom-Jordan identity, we recover the usual Jordan identity. Observe that the definition of the Hom-Jordan identity in [20] is slightly different from the one formerly given in [18].

Hom-Maltsev algebras are introduced in [20] in connection with a study of Hom-alternative algebras introduced in [18]. In fact it is proved ([20], Theorem 3.8) that every Homalternative algebra is Hom-Maltsev admissible; that is, the commutator Hom-algebra of any Hom-alternative algebra is a Hom-Maltsev algebra (this is the Hom-analogue of Maltsev's construction of Maltsev algebras as commutator algebras of alternative algebras [7]). This result is also mentioned in [16], Section 4, using an approach via Hom-Akivis algebras (this approach is close to the one of Maltsev in [7]). Also, every Hom-alternative algebra is Hom-Jordan admissible; that is, its plus Hom-algebra is a Hom-Jordan algebra ([20]). Examples of Hom-alternative algebras and Hom-Jordan algebras could be found in $[18,20]$. An example of a right Hom-alternative algebra that is not left Hom-alternative is given in [23].

Equivalent to (2) defining identities of Hom-Maltsev algebras are found in [20] where, in particular, it is shown that the identity

$$
\begin{gathered}
J_{\alpha}(\alpha(x), \alpha(y), w * z)+J_{\alpha}(\alpha(w), \alpha(y), x * z) \\
\quad=J_{\alpha}(x, y, z) * \alpha^{2}(w)+J_{\alpha}(w, y, z) * \alpha^{2}(x)
\end{gathered}
$$

is equivalent to (2) in any anticommutative Hom-algebra $(A, *, \alpha)([20]$, Proposition 2.7). In [34], it is proved that, in any anticommutative Hom-algebra $(A, *, \alpha)$, the HomMaltsev identity (2) is equivalent to

$$
\begin{aligned}
& J_{\alpha}(\alpha(x), \alpha(y), u * v) \\
& =\alpha^{2}(u) * J_{\alpha}(x, y, v)+J_{\alpha}(x, y, u) * \alpha^{2}(v) \\
& \quad-2 J_{\alpha}(\alpha(u), \alpha(v), x * y) .
\end{aligned}
$$

Therefore, apart from (2), identities (6) and (7) may be taken as defining identities of a Hom-Maltsev algebra.

The Hom-algebras mentioned above are binary Homalgebras. The first generalization of binary algebras was the ternary algebras introduced in [1]. Ternary algebraic structures also appeared in various domains of theoretical and mathematical physics (see, e.g., [35]). Likewise, binary Homalgebras are generalized to $n$-ary Hom-algebra structures in [11] (see also [21]).

Definition 6 (see [11]). A ternary Hom-Nambu algebra is a triple $(A,[,],, \alpha)$ in which $A$ is a $\mathbb{K}$-vector space, $[,]:, A \times$ $A \times A \rightarrow A$ is a trilinear map, and $\alpha=\left(\alpha_{1}, \alpha_{2}\right)$ is a pair of linear maps (the twisting maps) such that the identity

$$
\begin{aligned}
& {\left[\alpha_{1}(x), \alpha_{2}(y),[u, v, w]\right]} \\
& =\left[[x, y, u], \alpha_{1}(v), \alpha_{2}(w)\right] \\
& \quad+\left[\alpha_{1}(u),[x, y, v], \alpha_{2}(w)\right] \\
& \quad+\left[\alpha_{1}(u), \alpha_{2}(v),[x, y, w]\right]
\end{aligned}
$$

holds for all $u, v, w, x$, and $y$ in A. Identity (8) is called the ternary Hom-Nambu identity.

Remark 7. When $\alpha_{1}=$ id $=\alpha_{2}$ one recovers the usual ternary Nambu algebra. One may refer to [35] for the origins of Nambu algebras. In [11], examples of $n$-ary Hom-Nambu algebras that are not Nambu algebras are provided.

Definition 8 (see [21]). A Hom-Lie triple system (Hom-Lts) is a ternary Hom-algebra $\left(A,[,],, \alpha=\left(\alpha_{1}, \alpha_{2}\right)\right)$ such that

$$
\begin{aligned}
& {[x, y, z]=-[y, x, z],} \\
& \circlearrowleft_{x, y, z}[x, y, z]=0,
\end{aligned}
$$

and the ternary Hom-Nambu identity (8) holds in $\left(A,[,],, \alpha=\left(\alpha_{1}, \alpha_{2}\right)\right)$.

One notes that when the twisting maps $\alpha_{1}, \alpha_{2}$ are both equal to the identity map id, then we recover the usual notion of a Lie triple system $[2,3]$. Examples of Hom-Lts could be found in [21].

A particular situation, interesting for our setting, occurs when the twisting maps $\alpha_{i}$ are all equal, $\alpha_{1}=\alpha_{2}=\alpha$ and $\alpha([x, y, z])=[\alpha(x), \alpha(y), \alpha(z)]$ for all $x, y$, and $z$ in $A$. The Hom-Lie triple system $(A,[,],, \alpha)$ is then said to be multiplicative [21]. In case of multiplicativity, the ternary Hom-Nambu identity (8) then reads

$$
\begin{aligned}
& {[\alpha(x), \alpha(y),[u, v, w]]} \\
& \quad=[[x, y, u], \alpha(v), \alpha(w)] \\
& \quad+[\alpha(u),[x, y, v], \alpha(w)] \\
& \quad+[\alpha(u), \alpha(v),[x, y, w]] .
\end{aligned}
$$

In [14] a (multiplicative) Hom-triple system is defined as a (multiplicative) ternary Hom-algebra $(A,[,],, \alpha)$ such that (9) and (10) are satisfied (thus a multiplicative HomLts is seen as a Hom-triple system in which identity (11) holds; observe that this definition of a Hom-triple system is different from the one formerly given in [21], where a 
Hom-triple system is just the Hom-algebra $(A,[,],, \alpha))$. With this vision of a Hom-triple system, it is shown [14] that every multiplicative non-Hom-associative algebra (i.e., not necessarily Hom-associative algebra) has a natural Homtriple system structure if defining $[x, y, z]:=[[x, y], \alpha(z)]-$ as $(x, y, z)+\operatorname{as}(y, x, z)$. We note here that we get the same result if defining another ternary operation on a given Homalgebra. Specifically, we have the following result.

Proposition 9. Let $\mathscr{A}=(A, *, \alpha)$ be a multiplicative Homalgebra. Define on $\mathscr{A}$ the ternary operation

$$
(x, y, z):=a s^{J}(y, z, x)
$$

for all $x, y$, and $z \in A$. Then $(A,(,),, \alpha)$ is a multiplicative Hom-triple system.

Proof. A proof follows from the straightforward checking of identities (9) and (10) for "(, , )" using the commutativity of the Jordan product “o."

Since our results here depend on multiplicativity, in the rest of this paper we assume that all Hom-algebras (binary or ternary) are multiplicative and while dealing with the binary operation "*" and where there is no danger of confusion, we will use juxtaposition in order to reduce the number of braces; that is, for example, $x y * \alpha(z)$ means $(x * y) * \alpha(z)$.

Various results and constructions related to Hom-Lts are given in [21]. In particular, it is shown that every Lts $L$ can be twisted along any self-morphism of $L$ into a multiplicative Hom-Lts. For our purpose we just mention the following result.

Proposition 10 (see [21]). Let $(A, *)$ be a Maltsev algebra and $\alpha: A \rightarrow A$ an algebra morphism. Then $A_{\alpha}:=\left(A,[,,]_{\alpha}, \alpha\right)$ is a multiplicative Hom-Lts, where $[x, y, z]_{\alpha}=\alpha(2 x y * z-y z *$ $x-z x * y)$, for all $x, y$, and $z$ in $A$.

One observes that the product $[x, y, z]=2 x y * z-y z *$ $x-z x * y$ is the one defined in [6] providing a Maltsev algebra $(A, *)$ with a Lts structure. A construction describing another view of Proposition 10 above will be given in Section 3 (see Proposition 16) via Hom-Maltsev algebras. For the time being, we point out the following slight generalization of the result above, producing a sequence of multiplicative Hom-Lts from a given Maltsev algebra.

Proposition 11. Let $(A, *)$ be a Maltsev algebra and $\alpha: A \rightarrow$ $A$ an algebra morphism. Let $\alpha^{0}=i d$ and, for any integer $n \geq 1$, $\alpha^{n}=\alpha \circ \alpha^{n-1}$. If one defines on $A$ a trilinear operation $[,,]_{\alpha^{n}}$ by

$$
[x, y, z]_{\alpha^{n}}=\alpha^{n}(2 x y * z-y z * x-z x * y)
$$

for all $x, y$, and $z$ in $A$, then $\left(A,[,,]_{\alpha^{n}}, \alpha^{n}\right)$ is a multiplicative Hom-Lts.

Proof. Let $[x, y, z]=2 x y * z-y z * x-z x * y$ and then $[x, y, z]_{\alpha^{n}}=\alpha^{n}([x, y, z])$. We shall use the fact that $(A,[,]$, is a Lts [6]. Identities (9) and (10) for $[x, y, z]_{\alpha^{n}}$ are quite obvious. Next,

$$
\begin{aligned}
& {\left[\alpha^{n}(x), \alpha^{n}(y),[u, v, w]_{\alpha^{n}}\right]_{\alpha^{n}} } \\
&=\left[\alpha^{n}(x), \alpha^{n}(y), \alpha^{n}([u, v, w])\right]_{\alpha^{n}} \\
&=\alpha^{2 n}([x, y,[u, v, w]]) \\
&=\alpha^{2 n}([[x, y, u], v, w])+\alpha^{2 n}([u,[x, y, v], w]) \\
&+\alpha^{2 n}([u, v,[x, y, w]]) \\
&=\left[\alpha^{n}([x, y, u]), \alpha^{n}(v), \alpha^{n}(w)\right]_{\alpha^{n}} \\
&+ {\left[\alpha^{n}(u), \alpha^{n}([x, y, v]), \alpha^{n}(w)\right]_{\alpha^{n}} } \\
&+\left[\alpha^{n}(u), \alpha^{n}(v), \alpha^{n}([x, y, w])\right]_{\alpha^{n}} \\
&= {\left[[x, y, u]_{\alpha^{n}}, \alpha^{n}(v), \alpha^{n}(w)\right]_{\alpha^{n}} } \\
&+\left[\alpha^{n}(u),[x, y, v]_{\alpha^{n}}, \alpha^{n}(w)\right]_{\alpha^{n}} \\
&+\left[\alpha^{n}(u), \alpha^{n}(v),[x, y, w]_{\alpha^{n}}\right]_{\alpha^{n}}
\end{aligned}
$$

and so (11) holds for $[,,]_{\alpha^{n}}$. Thus $\left(A,[,,]_{\alpha^{n}}, \alpha^{n}\right)$ is a multiplicative Hom-Lts.

In [12] we defined a Hom-Bol algebra as a twisted generalization of a (left) Bol algebra. For the introduction and original studies of Bol algebras, we refer to [25-27] (the defining identities of left Bol algebras are recalled in Section 5 of the present paper). Bol algebras are further considered in, for example, $[29,30]$.

Definition 12 (see [12]). A Hom-Bol algebra is a quadruple $(A,[],,(,),, \alpha)$ in which $A$ is a vector space, "[, ]" a binary operation, "(, , )" a ternary operation on $A$, and $\alpha: A \rightarrow A$ a linear map such that

(HB1) $\alpha([x, y])=[\alpha(x), \alpha(y)]$.

(HB2) $\alpha((x, y, z))=(\alpha(x), \alpha(y), \alpha(z))$.

(HB3) $[x, y]=-[y, x]$.

(HB4) $(x, y, z)=-(y, x, z)$.

$(\mathrm{HB} 5) \circlearrowleft_{x, y, z}(x, y, z)=0$.

$(\mathrm{HB} 6)(\alpha(x), \alpha(y),[u, v])=\left[(x, y, u), \alpha^{2}(v)\right]+\left[\alpha^{2}(u)\right.$, $(x, y, v)]+(\alpha(u), \alpha(v),[x, y])-[[\alpha(u), \alpha(v)]$, $[\alpha(x), \alpha(y)]]$.

$\left(\right.$ HB7) $\left(\alpha^{2}(x), \alpha^{2}(y),(u, v, w)\right)=\left((x, y, u), \alpha^{2}(v), \alpha^{2}(w)\right)+$ $\left(\alpha^{2}(u),(x, y, v), \alpha^{2}(w)\right)+\left(\alpha^{2}(u), \alpha^{2}(v),(x, y, w)\right)$

for all $u, v, w, x, y$, and $z \in A$.

Identities (HB1) and (HB2) mean the multiplicativity of $(A,[],,(,),, \alpha)$. It is built into our definition for convenience.

One observes that for $\alpha=$ id identities (HB3)-(HB7) reduce to the defining identities of a (left) Bol algebra [25] (see also $[29,30])$. If $[x, y]=0$ for all $x, y \in A$, then $(A,[],,(,),, \alpha)$ becomes a (multiplicative) Hom-Lts $\left(A,(,),, \alpha^{2}\right)$. 
Construction results and some examples of Hom-Bol algebras are given in [12]. In particular, Hom-Bol algebras can be constructed from Maltsev algebras. The Hom-analogues of the construction of Bol algebras from Maltsev algebras [25] or from right alternative algebras [31] (see also [29]) are considered in this paper.

\section{Hom-Lts and Hom-Bol Algebras from Hom- Maltsev Algebras}

In this section, we prove that every multiplicative HomMaltsev algebra has a natural multiplicative Hom-Lts structure (Theorem 14) and, moreover, a natural Hom-Bol algebra structure (Theorem 17). Theorem 14 could be seen as the Hom-analogue of Loos' result ([6], Satz 1) although his proof cannot be reproduced here. Besides identities (6) and (7), Lemma 13 below is a tool in the proof of this result. Theorem 17 could be seen as the Hom-analogue of a construction by Mikheev [25] of Bol algebras from Maltsev algebras. Proposition 16 is another view of a result in [21] (see Proposition 10 above).

In his work [6], Loos considered in a Maltsev algebra $(A, *)$ the following ternary operation:

$$
\{x, y, z\}=2 x y * z-y z * x-z x * y .
$$

Then $(A,\{,\}$,$) turns out to be a Lts. This result, in the Hom-$ algebra setting, looks as in Theorem 14 below. Similarly as in the Loos construction, our investigations are based on the following ternary operation in a Hom-Maltsev algebra $(A, *, \alpha)$ :

$$
\{x, y, z\}_{\alpha}=2 x y * \alpha(z)-y z * \alpha(x)-z x * \alpha(y) .
$$

From (16) it clearly follows that $\{,,\}_{\alpha}$ can also be written as

$$
\{x, y, z\}_{\alpha}=-J_{\alpha}(x, y, z)+3 x y * \alpha(z) .
$$

One observes that when $\alpha=$ id, we recover product (15). First, we prove the following.

Lemma 13. Let $(A, *, \alpha)$ be a Hom-Maltsev algebra. If one defines on $(A, *, \alpha)$ a ternary operation " $\{,,\}_{\alpha}$ " by (16), then

$$
\begin{aligned}
\{\alpha(x), \alpha(y), u * v\}_{\alpha}= & \alpha^{2}(u) *\{x, y, v\}_{\alpha} \\
& +\{x, y, u\}_{\alpha} * \alpha^{2}(v) \\
& -J_{\alpha}(\alpha(u), \alpha(v), x * y)
\end{aligned}
$$

for all $u, v, x$, and $y$ in $A$.

Proof. Let us write (7) as

$$
\begin{aligned}
-J_{\alpha} & (\alpha(x), \alpha(y), u * v) \\
= & -J_{\alpha}(x, y, u) * \alpha^{2}(v)+\alpha^{2}(u) *\left(-J_{\alpha}(x, y, v)\right) \\
& +3 J_{\alpha}(\alpha(u), \alpha(v), x * y) \\
& -J_{\alpha}(\alpha(u), \alpha(v), x * y) .
\end{aligned}
$$

That is,

$$
\begin{aligned}
-J_{\alpha} & (\alpha(x), \alpha(y), u * v) \\
= & -J_{\alpha}(x, y, u) * \alpha^{2}(v)+\alpha^{2}(u) *\left(-J_{\alpha}(x, y, v)\right) \\
& +3 \alpha(u) \alpha(v) * \alpha(x * y)+3(\alpha(v) * x y) \\
& * \alpha^{2}(u)+3(x y * \alpha(u)) * \alpha^{2}(v) \\
& -J_{\alpha}(\alpha(u), \alpha(v), x * y) .
\end{aligned}
$$

Therefore, by multiplicativity, we have

$$
\begin{aligned}
-J_{\alpha} & (\alpha(x), \alpha(y), u * v)+3 \alpha(x) \alpha(y) * \alpha(u * v) \\
= & \left(-J_{\alpha}(x, y, u)+3 x y * \alpha(u)\right) * \alpha^{2}(v)+\alpha^{2}(u) \\
& *\left(-J_{\alpha}(x, y, v)+3 x y * \alpha(v)\right) \\
& -J_{\alpha}(\alpha(u), \alpha(v), x * y)
\end{aligned}
$$

and so, we get (18) by (17).

We now prove the following.

Theorem 14. Let $(A, *, \alpha)$ be a multiplicative Hom-Maltsev algebra. If one defines on $(A, *, \alpha)$ a ternary operation " $\{,\}_{\alpha}$ " by (16), then $\left(A,\{,\}_{\alpha}, \alpha^{2}\right)$ is a multiplicative Hom-Lts.

Proof. We must prove the validity of (9), (10), and (11) for operation (16) in the Hom-Maltsev algebra $(A, *, \alpha)$.

First observe that the multiplicativity of $(A, *, \alpha)$ implies that $\alpha^{2}\left(\{x, y, z\}_{\alpha}\right)=\left\{\alpha^{2}(x), \alpha^{2}(y), \alpha^{2}(z)\right\}_{\alpha}$, with $x, y$, and $z$ in $A$.

From the skew-symmetry of " $*$ " and $J_{\alpha}(x, y, z)$, it clearly follows from (17) that $\{x, y, z\}_{\alpha}=-\{y, x, z\}_{\alpha}$ which is (9) for " $\{,,\}_{\alpha}$ "”

Next, using (17) and the skew-symmetry of $J_{\alpha}(x, y, z)$ where applicable, we compute

$$
\begin{aligned}
& \{x, y, z\}_{\alpha}+\{y, z, x\}_{\alpha}+\{z, x, y\}_{\alpha} \\
& =-J_{\alpha}(x, y, z)+3 x y * \alpha(z)-J_{\alpha}(y, z, x)+3 y z \\
& \quad * \alpha(x)-J_{\alpha}(z, x, y)+3 z x * \alpha(y) \\
& =-3 J_{\alpha}(x, y, z)+3 J_{\alpha}(x, y, z)=0
\end{aligned}
$$

and thus $\sigma_{x, y, z}\{x, y, z\}_{\alpha}=0$, so we get (10) for " $\{,,\}_{\alpha}$." Then

Consider now $\left\{\alpha^{2}(x), \alpha^{2}(y),\{u, v, w\}_{\alpha}\right\}_{\alpha}$ in $(A, *, \alpha)$.

$$
\begin{gathered}
\left\{\alpha^{2}(x), \alpha^{2}(y),\{u, v, w\}_{\alpha}\right\}_{\alpha}=\left\{\alpha^{2}(x), \alpha^{2}(y), 2 u v\right. \\
* \alpha(w)-v w * \alpha(u)-w u * \alpha(v)\}_{\alpha} \quad(\text { by }(16)) \\
=\left\{\alpha^{2}(x), \alpha^{2}(y), 2 u v * \alpha(w)\right\}_{\alpha}-\left\{\alpha^{2}(x), \alpha^{2}(y),\right. \\
v w * \alpha(u)\}_{\alpha}-\left\{\alpha^{2}(x), \alpha^{2}(y), w u * \alpha(v)\right\}_{\alpha} \\
=\{\alpha(x), \alpha(y), 2 u * v\}_{\alpha} * \alpha^{3}(w)+\alpha^{2}(2 u * v)
\end{gathered}
$$




$$
\begin{aligned}
& *\{\alpha(x), \alpha(y), \alpha(w)\}_{\alpha}-J_{\alpha}\left(\alpha(2 u * v), \alpha^{2}(w),\right. \\
& \alpha(x * y))-\{\alpha(x), \alpha(y), v * w\}_{\alpha} * \alpha^{3}(u) \\
& -\alpha^{2}(v * w) *\{\alpha(x), \alpha(y), \alpha(u)\}_{\alpha}+J_{\alpha}(\alpha(v \\
& \left.* w), \alpha^{2}(u), \alpha(x * y)\right)-\{\alpha(x), \alpha(y), w * u\}_{\alpha} \\
& * \alpha^{3}(v)-\alpha^{2}(w * u) *\{\alpha(x), \alpha(y), \alpha(v)\}_{\alpha} \\
& +J_{\alpha}\left(\alpha(w * u), \alpha^{2}(v), \alpha(x * y)\right) \quad(\text { by }(18)) \\
& =\left(2\{x, y, u\}_{\alpha} * \alpha^{2}(v)+2 \alpha^{2}(u) *\{x, y, v\}_{\alpha}\right. \\
& \left.-2 J_{\alpha}(\alpha(u), \alpha(v), x * y)\right) * \alpha^{3}(w)+2 \alpha^{2}(u * v) \\
& *\{\alpha(x), \alpha(y), \alpha(w)\}_{\alpha}-J_{\alpha}\left(\alpha(2 u * v), \alpha^{2}(w),\right. \\
& \alpha(x * y))-\left(\{x, y, v\}_{\alpha} * \alpha^{2}(w)+\alpha^{2}(v)\right. \\
& \left.*\{x, y, w\}_{\alpha}-J_{\alpha}(\alpha(v), \alpha(w), x * y)\right) * \alpha^{3}(u) \\
& -\alpha^{2}(v * w) *\{\alpha(x), \alpha(y), \alpha(u)\}_{\alpha}+J_{\alpha}(\alpha(v \\
& \left.* w), \alpha^{2}(u), \alpha(x * y)\right)-\left(\{x, y, w\}_{\alpha} * \alpha^{2}(u)\right. \\
& \left.+\alpha^{2}(w) *\{x, y, u\}_{\alpha}-J_{\alpha}(\alpha(w), \alpha(u), x * y)\right) \\
& * \alpha^{3}(v)-\alpha^{2}(w * u) *\{\alpha(x), \alpha(y), \alpha(v)\}_{\alpha} \\
& +J_{\alpha}\left(\alpha(w * u), \alpha^{2}(v), \alpha(x * y)\right)
\end{aligned}
$$$$
\text { (again by (18)) }
$$$$
=2\{x, y, u\}_{\alpha} \alpha^{2}(v) * \alpha^{3}(w)+2 \alpha^{2}(u)\{x, y, v\}_{\alpha}
$$$$
* \alpha^{3}(w)-2 J_{\alpha}(\alpha(u), \alpha(v), x * y) * \alpha^{3}(w)
$$$$
+2 \alpha^{2}(u * v) *\{\alpha(x), \alpha(y), \alpha(w)\}_{\alpha}-J_{\alpha}(\alpha(2 u
$$$$
\left.* v), \alpha^{2}(w), \alpha(x * y)\right)-\{x, y, v\}_{\alpha} \alpha^{2}(w) * \alpha^{3}(u)
$$$$
-\alpha^{2}(v)\{x, y, w\}_{\alpha} * \alpha^{3}(u)+J_{\alpha}(\alpha(v), \alpha(w), x
$$$$
* y) * \alpha^{3}(u)-\alpha^{2}(v * w) *\{\alpha(x), \alpha(y), \alpha(u)\}_{\alpha}
$$$$
+J_{\alpha}\left(\alpha(v * w), \alpha^{2}(u), \alpha(x * y)\right)-\{x, y, w\}_{\alpha}
$$$$
\cdot \alpha^{2}(u) * \alpha^{3}(v)-\alpha^{2}(w)\{x, y, u\}_{\alpha} * \alpha^{3}(v)
$$$$
+J_{\alpha}(\alpha(w), \alpha(u), x * y) * \alpha^{3}(v)-\alpha^{2}(w * u)
$$$$
*\{\alpha(x), \alpha(y), \alpha(v)\}_{\alpha}+J_{\alpha}\left(\alpha(w * u), \alpha^{2}(v), \alpha(x\right.
$$$$
* y))=2\{x, y, u\}_{\alpha} \alpha^{2}(v) * \alpha^{3}(w)-\alpha^{2}(v * w)
$$$$
* \alpha\left(\{x, y, u\}_{\alpha}\right)-\alpha^{2}(w)\{x, y, u\}_{\alpha} * \alpha^{3}(v)
$$$$
+2 \alpha^{2}(u)\{x, y, v\}_{\alpha} * \alpha^{3}(w)-\{x, y, v\}_{\alpha} \alpha^{2}(w)
$$

$$
\begin{aligned}
& * \alpha^{3}(u)-\alpha^{2}(w * u) * \alpha\left(\{x, y, v\}_{\alpha}\right)+2 \alpha^{2}(u \\
& * v) * \alpha\left(\{x, y, w\}_{\alpha}\right)-\alpha^{2}(v)\{x, y, w\}_{\alpha} * \alpha^{3}(u) \\
& -\{x, y, w\}_{\alpha} \alpha^{2}(u) * \alpha^{3}(v)-2 J_{\alpha}(\alpha(u), \alpha(v), x \\
& * y) * \alpha^{3}(w)-J_{\alpha}\left(\alpha(2 u * v), \alpha^{2}(w), \alpha(x * y)\right) \\
& +J_{\alpha}(\alpha(v), \alpha(w), x * y) * \alpha^{3}(u)+J_{\alpha}(\alpha(v * w), \\
& \left.\alpha^{2}(u), \alpha(x * y)\right)+J_{\alpha}(\alpha(w), \alpha(u), x * y) \\
& * \alpha^{3}(v)+J_{\alpha}\left(\alpha(w * u), \alpha^{2}(v), \alpha(x * y)\right)
\end{aligned}
$$

(rearranging terms)

$$
\begin{aligned}
= & \left\{\{x, y, u\}_{\alpha}, \alpha^{2}(v), \alpha^{2}(w)\right\}_{\alpha}+\left\{\alpha^{2}(u),\{x, y, v\}_{\alpha},\right. \\
& \left.\alpha^{2}(w)\right\}_{\alpha}+\left\{\alpha^{2}(u), \alpha^{2}(v),\{x, y, w\}_{\alpha}\right\}_{\alpha} \\
& +\left[-2\left(J_{\alpha}(\alpha(u), \alpha(v), x * y) * \alpha^{3}(w)\right.\right. \\
& \left.+J_{\alpha}\left(\alpha(u * v), \alpha^{2}(w), \alpha(x * y)\right)\right) \\
& +J_{\alpha}(\alpha(v), \alpha(w), x * y) * \alpha^{3}(u) \\
& +J_{\alpha}\left(\alpha(v * w), \alpha^{2}(u), \alpha(x * y)\right) \\
& +J_{\alpha}(\alpha(w), \alpha(u), x * y) * \alpha^{3}(v) \\
& \left.+J_{\alpha}\left(\alpha(w * u), \alpha^{2}(v), \alpha(x * y)\right)\right] .
\end{aligned}
$$

In this latest expression, denote by $N(u, v, w, x, y)$ the expression in "[…"; to conclude, we proceed to show that $N(u, v, w, x, y)=0$.

Observe first that, by (6), we have

$$
\begin{aligned}
J_{\alpha}(\alpha(u), x * y, \alpha(w)) * \alpha^{2}(\alpha(v)) \\
\quad+J_{\alpha}(\alpha(v), x * y, \alpha(w)) * \alpha^{2}(\alpha(u)) \\
=J_{\alpha}\left(\alpha^{2}(u), \alpha(x * y), \alpha(v) * \alpha(w)\right) \\
\quad+J_{\alpha}\left(\alpha^{2}(v), \alpha(x * y), \alpha(u) * \alpha(w)\right) .
\end{aligned}
$$

That is,

$$
\begin{aligned}
J_{\alpha}(\alpha(w), \alpha(u), x * y) * \alpha^{3}(v) \\
\quad+J_{\alpha}\left(\alpha(w * u), \alpha^{2}(v), \alpha(x * y)\right) \\
=J_{\alpha}\left(\alpha(v * w), \alpha^{2}(u), \alpha(x * y)\right) \\
\quad+J_{\alpha}(\alpha(v), \alpha(w), x * y) * \alpha^{3}(u) .
\end{aligned}
$$

With this observation, the expression $N(u, v, w, x, y)$ is transformed as follows:

$$
\begin{aligned}
& N(u, v, w, x, y)=2\left[-J_{\alpha}(\alpha(u), \alpha(v), x * y)\right. \\
& \left.\quad * \alpha^{3}(w)-J_{\alpha}\left(\alpha(u * v), \alpha^{2}(w), \alpha(x * y)\right)\right]
\end{aligned}
$$




$$
\begin{aligned}
& +2\left[J_{\alpha}\left(\alpha(v * w), \alpha^{2}(u), \alpha(x * y)\right)\right. \\
& \left.+J_{\alpha}(\alpha(v), \alpha(w), x * y) * \alpha^{3}(u)\right] \\
& =2\left[-J_{\alpha}\left(\alpha^{2}(w), \alpha(x * y), \alpha(u) * \alpha(v)\right)\right. \\
& -J_{\alpha}\left(\alpha^{2}(u), \alpha(x * y), \alpha(w) * \alpha(v)\right) \\
& -J_{\alpha}(\alpha(u), \alpha(v), x * y) * \alpha^{3}(w) \\
& \left.+J_{\alpha}(\alpha(v), \alpha(w), x * y) * \alpha^{3}(u)\right] \\
& =2\left[-J_{\alpha}(\alpha(w), x * y, \alpha(v)) * \alpha^{3}(u)\right. \\
& -J_{\alpha}(\alpha(u), x * y, \alpha(v)) * \alpha^{3}(w) \\
& -J_{\alpha}(\alpha(u), \alpha(v), x * y) * \alpha^{3}(w) \\
& \left.+J_{\alpha}(\alpha(v), \alpha(w), x * y) * \alpha^{3}(u)\right] \\
& (\text { applying }(6) \text { to } \\
& -J_{\alpha}\left(\alpha^{2}(w), \alpha(x * y), \alpha(u) * \alpha(v)\right) \\
& \left.-J_{\alpha}\left(\alpha^{2}(u), \alpha(x * y), \alpha(w) * \alpha(v)\right)\right) \\
& =0\left(\text { by the skew-symmetry of } J_{\alpha}(x, y, z)\right) .
\end{aligned}
$$

Therefore, we obtain that (11) holds for " $\{,,\}_{\alpha}$ " and we conclude that $\left(A,\{,,\}_{\alpha}, \alpha^{2}\right)$ is a Hom-Lts.

Remark 15. In the proof of his result, Loos ([6], Satz 1) used essentially the fact that the left translations $L(x)$ in a Maltsev algebra $(A, *)$ are derivations with respect to the ternary operation " $\{,$,$\} " defined by (15). Unfortunately, for Hom-$ Maltsev algebras such a tool is still not available at hand.

From [20] (Theorem 2.12) we know that any Maltsev algebra $A$ can be twisted into a Hom-Maltsev algebra along any linear self-map of $A$. Consistent with this result, we recall the following method for constructing Hom-Lts which, in fact, is a result in [21] (see also Propositions 10 and 11 above) but using a Hom-Maltsev algebra construction in our proof (as a consequence of Theorem 14).

Proposition 16. Let $(A, *)$ be a Maltsev algebra and $\alpha$ any selfmorphism of $(A, *)$. If one defines on $(A, *)$ a ternary operation “ $\{,\}_{\alpha}$ ” by

$$
\{x, y, z\}_{\alpha}=\alpha^{2}(2 x y * z-y z * x-z x * y),
$$

then $\left(A,\{,\}_{\alpha}, \alpha^{2}\right)$ is a multiplicative Hom-Lts.

Proof. One knows ([20], Theorem 2.12) that, from $(A, *)$ and any self-morphism $\alpha$ of $(A, *)$, we get a (multiplicative) HomMaltsev algebra $(A, \widetilde{*}, \alpha)$, where $x \widetilde{*} y=\alpha(x * y)$ for all $x, y$ in $A$. Next, if one defines on $(A, \widetilde{*}, \alpha)$ a ternary operation

$$
\begin{aligned}
\{x, y, z\}_{\alpha}:= & 2(x \widetilde{*}) \widetilde{*} \alpha(z)-(y \widetilde{*}) \widetilde{*} \alpha(x) \\
& -(z \widetilde{*} x) \widetilde{*} \alpha(y),
\end{aligned}
$$

then, by Theorem $14,\left(A,\{,,\}_{\alpha}, \alpha^{2}\right)$ is a Hom-Lts and " $\{,,\}_{\alpha}$ " is expressed through “*” as

$$
\begin{aligned}
\{x, y, z\}_{\alpha}= & 2 \alpha(\alpha(x * y) * \alpha(z)) \\
& -\alpha(\alpha(y * z) * \alpha(x)) \\
& -\alpha(\alpha(z * x) * \alpha(y)) \\
= & 2 \alpha^{2}(x y * z)-\alpha^{2}(y z * x)-\alpha^{2}(z x * y) \\
= & \alpha^{2}(2 x y * z-y z * x-z x * y) .
\end{aligned}
$$

Observe that though constructed in quite a different way, the operation " $\{,,\}_{\alpha}$ " in Proposition 16 above coincides with "[, , ] $]_{\alpha^{n}}$ " in Proposition 11 for $n=2$.

Combining Lemma 13 and Theorem 14, we get the following result.

Theorem 17. Let $(A, *, \alpha)$ be a multiplicative Hom-Maltsev algebra. If one defines on $(A, *, \alpha)$ a ternary operation $(,,)_{\alpha}$ by

$$
(x, y, z)_{\alpha}:=\frac{1}{3}\{x, y, z\}_{\alpha},
$$

where " $\{,\}_{\alpha}$ " is defined by (17), then $\left(A, *,(,,)_{\alpha}, \alpha\right)$ is a HomBol algebra.

Proof. Definition (30) and Theorem 14 imply that $\left(A,(,,)_{\alpha}\right.$, $\alpha^{2}$ ) is a multiplicative Hom-Lts; that is, (HB4), (HB5), and (HB7) hold for $\left(A, *,(,)_{\alpha}, \alpha\right)$. Now, (HB1), (HB2), and (HB3) are, respectively, the multiplicativity and skew-symmetry of “*”; next, we are done if we prove (HB6) for $\left(A, *,(,)_{\alpha}, \alpha\right)$.

From (17) and multiplicativity we have

$$
\begin{aligned}
-J_{\alpha} & (\alpha(u), \alpha(v), x * y) \\
= & \{\alpha(u), \alpha(v), x * y\}_{\alpha}-3(\alpha(u) \alpha(v)) \\
& *(\alpha(x) \alpha(y))
\end{aligned}
$$

and then (18) takes the form

$$
\begin{aligned}
& \{\alpha(x), \alpha(y), u * v\}_{\alpha} \\
& =\{x, y, u\}_{\alpha} * \alpha^{2}(v)+\alpha^{2}(u) *\{x, y, v\}_{\alpha} \\
& \quad+\{\alpha(u), \alpha(v), x * y\}_{\alpha}-3(\alpha(u) \alpha(v)) \\
& \quad *(\alpha(x) \alpha(y)) .
\end{aligned}
$$

Multiplying by $1 / 3$ each member of this latter equality and using (30), we get

$$
\begin{aligned}
(\alpha(x) & , \alpha(y), u * v)_{\alpha} \\
= & (x, y, u)_{\alpha} * \alpha^{2}(v)+\alpha^{2}(u) *(x, y, v)_{\alpha} \\
& +(\alpha(u), \alpha(v), x * y)_{\alpha}-(\alpha(u) \alpha(v)) \\
& *(\alpha(x) \alpha(y))
\end{aligned}
$$


which is (HB6) for $\left(A, *,(,)_{\alpha}, \alpha\right)$. So $\left(A, *,(,)_{\alpha}, \alpha\right)$ is a Hom-Bol algebra.

Example 18. Let $A$ be a vector space with basis $\left\{e_{1}, e_{2}, e_{3}, e_{4}\right\}$. From [20] (Example 2.13) we know that if one considers the linear map $\alpha: A \rightarrow A$ given by

$$
\begin{aligned}
& \alpha\left(e_{1}\right)=e_{1}+e_{3} ; \\
& \alpha\left(e_{2}\right)=2 e_{2}+2 e_{4} ; \\
& \alpha\left(e_{3}\right)=-e_{3} ; \\
& \alpha\left(e_{4}\right)=-2 e_{4}
\end{aligned}
$$

and the multiplication table given by

$$
\begin{aligned}
& e_{1} * e_{2}=-2 e_{2}-2 e_{4}\left(=-e_{2} * e_{1}\right) ; \\
& e_{1} * e_{3}=e_{3}\left(=-e_{3} * e_{1}\right) ; \\
& e_{1} * e_{4}=-2 e_{4}\left(=-e_{4} * e_{1}\right) ; \\
& e_{2} * e_{3}=-4 e_{4}\left(=-e_{3} * e_{2}\right)
\end{aligned}
$$

(only nonzero products are specified), then $(A, *, \alpha)$ is a multiplicative Hom-Maltsev algebra. It is observed that $(A, *, \alpha)$ is not a Hom-Lie algebra nor a Maltsev algebra.

Now, by (17) and (30), one checks that the only nonzero ternary products $(x, y, z)_{\alpha}$ on $A$ with respect to the basis elements are

$$
\begin{aligned}
& \left(e_{1}, e_{2}, e_{1}\right)_{\alpha}=-4 e_{2}\left(=-\left(e_{2}, e_{1}, e_{1}\right)_{\alpha}\right) ; \\
& \left(e_{1}, e_{3}, e_{1}\right)_{\alpha}=-e_{3}\left(=-\left(e_{3}, e_{1}, e_{1}\right)_{\alpha}\right) ; \\
& \left(e_{1}, e_{4}, e_{1}\right)_{\alpha}=-4 e_{4}\left(=-\left(e_{4}, e_{1}, e_{1}\right)_{\alpha}\right) .
\end{aligned}
$$

By Theorem 17 we get that $\left(A, *,(,,)_{\alpha}, \alpha\right)$ is a Hom-Bol algebra.

Since any Hom-alternative algebra is Hom-Maltsev admissible ([20], Theorem 3.8), from Theorem 17 we have the following.

Corollary 19. Let $(A, *, \alpha)$ be a multiplicative Hom-alternative algebra. Then $\left(A,[],,(,,)_{\alpha}, \alpha\right)$ is a Hom-Bol algebra, where $(x, y, z)_{\alpha}:=-(1 / 3)(2[[x, y], \alpha(z)]-[[y, z], \alpha(x)]-$ $[[z, x], \alpha(y)])$, for all $x, y$, and $z \in A$.

The aim of Section 4 is a generalization of Corollary 19 to multiplicative right (or left) Hom-alternative algebras.

Various constructions of Hom-Lts are offered in [21] starting from either Hom-associative algebras, Hom-Lie algebras, Hom-Jordan triple systems, ternary totally Hom-associative algebras, Maltsev algebras, or alternative algebras. In practice, it is easier to construct Hom-Lts or Hom-Bol algebras from well-known (binary) algebras such as alternative algebras or Maltsev algebras. From this point of view, our construction results (Theorem 14, Proposition 16, and Theorem 17) have rather a theoretical feature (the extension to Hom-algebra setting of Loos' result [6] and a result by Mikheev [25]) than a practical method for constructing Hom-Lts or Hom-Bol algebras. However, it could be of some interest to get a HomLts or a Hom-Bol algebra from a given Hom-Maltsev algebra without resorting to the corresponding Maltsev algebra.

\section{Hom-Lts and Hom-Bol Algebras from Right (or Left) Hom-Alternative Algebras}

In this section we prove that every multiplicative right (or left) Hom-alternative algebra has a natural Hom-Bol algebra structure (and, subsequently, a natural Hom-Lts structure). This is the Hom-analogue of a result by Mikheev [31] and by Hentzel and Peresi [29] although with a different scheme of proof.

First we recall some few basic properties of right Homalternative algebras that could be found in $[18,23]$.

The linearized form of the right Hom-alternative identity as $(x, y, y)=0$ is given by the following result.

Lemma 20 (see [18]). If $(A, *, \alpha)$ is a Hom-algebra, then the following statements are equivalent.

(i) $(A, *, \alpha)$ is right Hom-alternative.

(ii) $(A, *, \alpha)$ satisfies

$$
\text { as }(x, y, z)=-a s(x, z, y)
$$

for all $x, y$, and $z \in A$.

(iii) $(A, *, \alpha)$ satisfies

$$
\alpha(x) *(y z+z y)=x y * \alpha(z)+x z * \alpha(y)
$$

for all $x, y$, and $z \in A$.

Observe that if $(A, *, \alpha)$ is a right Hom-alternative algebra, then $\left(A, *^{\mathrm{op}}, \alpha\right)$ is a left Hom-alternative algebra, where $x *{ }^{\mathrm{op}} y:=y * x$. So the mirrors of (37) and (38) hold for $\left(A, *^{\mathrm{op}}, \alpha\right)$ :

$$
\begin{aligned}
& \text { as }(x, y, z)=- \text { as }(y, x, z), \\
& \left(\left(x *^{\mathrm{op}} y\right)+\left(y *^{\mathrm{op}} x\right)\right) *^{\mathrm{op}} \alpha(z) \\
& \quad=\alpha(x) *^{\mathrm{op}}\left(y *^{\mathrm{op}} z\right)+\alpha(y) *^{\mathrm{op}}\left(x *^{\mathrm{op}} z\right) .
\end{aligned}
$$

Now we have the following.

Lemma 21. In any multiplicative right Hom-alternative alge$\operatorname{bra}(A, *, \alpha)$, the identity

$$
\begin{aligned}
\operatorname{as}( & {[u, v], \alpha(x), \alpha(y)) } \\
= & {\left[a s(u, x, y), \alpha^{2}(v)\right]+\left[\alpha^{2}(u), a s(v, x, y)\right] } \\
& +a s(\alpha(v), \alpha(u),[x, y]) \\
& -a s(\alpha(u), \alpha(v),[x, y])
\end{aligned}
$$

holds for all $x, y$, and $z \in A$. 
Proof. The identity

$$
\begin{aligned}
\text { as }(u v, \alpha(x), \alpha(y))= & \operatorname{as}(u, x, y) \alpha^{2}(v) \\
& +\alpha^{2}(u) \text { as }(v, x, y) \\
& -\operatorname{as}(\alpha(u), \alpha(v),[x, y])
\end{aligned}
$$

holds in any right Hom-alternative algebra (see [23], Theorem 7.1 (7.1.1c)). Next, in this identity, switching $u$ and $v$, we have

$$
\begin{aligned}
\text { as }(v u, \alpha(x), \alpha(y))= & \operatorname{as}(v, x, y) \alpha^{2}(u) \\
& +\alpha^{2}(v) \text { as }(u, x, y) \\
& -\operatorname{as}(\alpha(v), \alpha(u),[x, y]) .
\end{aligned}
$$

Then, subtracting memberwise this latter equality from the one above and using the linearity of as, we get (41).

Note that in the case when $(A, *, \alpha)$ is a left Homalternative algebra, identity (41) reads as

$$
\begin{aligned}
\text { as } & (\alpha(x), \alpha(y),[u, v]) \\
= & {\left[\operatorname{as}(x, y, u), \alpha^{2}(v)\right]+\left[\alpha^{2}(u), \text { as }(x, y, v)\right] } \\
& +\operatorname{as}([x, y], \alpha(v), \alpha(u)) \\
& \quad-\operatorname{as}([x, y], \alpha(u), \alpha(v)) .
\end{aligned}
$$

In any multiplicative right (or left) Hom-alternative algebra $(A, *, \alpha)$ we consider the ternary operation defined by (12); that is,

$$
(x, y, z):=\operatorname{as}^{J}(y, z, x),
$$

where as ${ }^{J}$ is the Hom-Jordan associator defined in Section 2. Observe that for $\alpha=$ id the ternary operation " $(,$,$) " is$ precisely the one defined in [29] (see also [31], Remark 2) and that makes any right (or left) alternative algebra into a left Bol algebra. In [29], Hentzel and Peresi used the approach of Mikheev [31] who formerly proved that the commutator algebra of any right alternative algebra has a left Bol algebra structure.

Proposition 22. (i) If $(A, *, \alpha)$ is a multiplicative right Homalternative algebra, then

$$
(x, y, z)=[[x, y], \alpha(z)]-2 a s(z, x, y)
$$

for all $x, y$, and $z \in A$.

(ii) If $(A, *, \alpha)$ is a multiplicative left Hom-alternative algebra, then

$$
(x, y, z)=[[x, y], \alpha(z)]-2 a s(x, y, z)
$$

for all $x, y$, and $z \in A$.

Proof. (i) From (12) we have

$$
\begin{aligned}
(x, y, z)= & (y \circ z) \circ \alpha(x)-\alpha(y) \circ(z \circ x) \\
= & ((y * z)+(z * y)) * \alpha(x) \\
& +[\alpha(x) *((y * z)+(z * y))]
\end{aligned}
$$

$$
\begin{aligned}
& -[\alpha(y) *((z * x)+(x * z))] \\
& -((z * x)+(x * z)) * \alpha(y) \\
& =((y * z)+(z * y)) * \alpha(x) \\
& +[(x * y) * \alpha(z)+(x * z) * \alpha(y)] \\
& -[(y * z) * \alpha(x)+(y * x) * \alpha(z)] \\
& -((z * x)+(x * z)) * \alpha(y) \quad(\text { by }(38)) \\
& =(z * y) * \alpha(x)+(x * y) * \alpha(z) \\
& -(y * x) * \alpha(z)-(z * x) * \alpha(y) \\
& =(z * y) * \alpha(x)-(z * x) * \alpha(y)+[x, y] \\
& * \alpha(z) \\
& =(z * y) * \alpha(x)-(z * x) * \alpha(y) \\
& +[[x, y], \alpha(z)]+\alpha(z) *[x, y] \\
& =[[x, y], \alpha(z)]+(z * y) * \alpha(x)-\alpha(z) \\
& *(y * x)-(z * x) * \alpha(y)+\alpha(z) \\
& *(x * y) \\
& =[[x, y], \alpha(z)]+\operatorname{as}(z, y, x)-\operatorname{as}(z, x, y) \\
& =[[x, y], \alpha(z)]-2 \operatorname{as}(z, x, y) \quad(\text { by }(37))
\end{aligned}
$$

and so we get (46).

(ii) Proceeding as above, but using (40) and then (39), one gets (47).

We are now in a position to prove the main result of this section.

Theorem 23. Let $(A, *, \alpha)$ be a multiplicative right (resp., left) Hom-alternative algebra. If one defines on $A$ a ternary operation “(, , )" by (46) (resp., (47)), then $\left(A,(,),, \alpha^{2}\right)$ is a Hom-Lts and $(A,[],,(,),, \alpha)$ is a Hom-Bol algebra.

Proof. We prove the theorem for a multiplicative right Homalternative algebra $(A, *, \alpha)$ (the proof of the left case is the mirror of the right one).

Identities (HB1) and (HB2) follow from the multiplicativity of $(A, *, \alpha)$. Identities (HB3) and (HB4) are obvious from the definition of "[, ]" and "(, , )"; identity (HB5) follows from Proposition 9.

In [22] Yau showed that if, on a multiplicative HomJordan algebra $(A, \circ, \alpha)$, define a ternary operation by

$$
[x, y, z]:=2(\alpha(x) \circ(y \circ z)-\alpha(y) \circ(x \circ z)),
$$

then $\left(A,[,],, \alpha^{2}\right)$ is a multiplicative Hom-Lts (see [22], Corollary 4.1). Now, observe that $[x, y, z]=2 \operatorname{as}^{J}(y, z, x)$; that is, $[x, y, z]=2(x, y, z)$. Therefore, since every multiplicative right Hom-alternative algebra is Hom-Jordan admissible 
(see [23], Theorem 4.3), we conclude that $\left(A,(,),, \alpha^{2}\right)$ is a multiplicative Hom-Lts and so identity (HB7) holds for $(A,[],,(,),, \alpha)$.

Next, $(A,[],,(,),, \alpha)$ is a Hom-Bol algebra if we prove that (HB6) additionally holds.

Write (46) as

$$
-2 \operatorname{as}(z, x, y)=(x, y, z)-[[x, y], \alpha(z)] .
$$

Multiplying each member of (41) by -2 and next using (50), we get

$$
\begin{aligned}
& (\alpha(x), \alpha(y),[u, v])-[[\alpha(x), \alpha(y)], \alpha([u, v])] \\
& =\left[(x, y, u)-[[x, y], \alpha(u)], \alpha^{2}(v)\right] \\
& \quad+\left[\alpha^{2}(u),(x, y, v)-[[x, y], \alpha(v)]\right] \\
& \quad+(\alpha(u),[x, y], \alpha(v)) \\
& \quad-\left[[\alpha(u),[x, y]], \alpha^{2}(v)\right] \\
& \quad-(\alpha(v),[x, y], \alpha(u)) \\
& \quad+\left[[\alpha(v),[x, y]], \alpha^{2}(u)\right] .
\end{aligned}
$$

That is,

$$
\begin{aligned}
(\alpha(x), \alpha(y),[u, v])= & {\left[(x, y, u), \alpha^{2}(v)\right] } \\
& +\left[\alpha^{2}(u),(x, y, v)\right] \\
& -([x, y], \alpha(u), \alpha(v)) \\
& +([x, y], \alpha(v), \alpha(u)) \\
& +\alpha([[x, y],[u, v]]) .
\end{aligned}
$$

Observe that

$$
\begin{aligned}
- & ([x, y], \alpha(u), \alpha(v))+([x, y], \alpha(v), \alpha(u)) \\
& =(\alpha(u),[x, y], \alpha(v))+([x, y], \alpha(v), \alpha(u)) \\
& =-(\alpha(v), \alpha(u),[x, y])
\end{aligned}
$$

$\left(\right.$ since $\sigma_{a, b, c}(a, b, c)=0$ by $\left.(\mathrm{HB} 5)\right)=(\alpha(u), \alpha(v),[x, y])$.

Therefore, (52) now reads

$$
\begin{aligned}
(\alpha(x), \alpha(y),[u, v])= & {\left[(x, y, u), \alpha^{2}(v)\right] } \\
& +\left[\alpha^{2}(u),(x, y, v)\right] \\
& +(\alpha(u), \alpha(v),[x, y]) \\
& -\alpha([[u, v],[x, y]])
\end{aligned}
$$

and so (HB6) holds for $(A,[],,(,),, \alpha)$. Thus we conclude that $(A,[],,(,),, \alpha)$ is a Hom-Bol algebra. One gets the same result in the case when $(A, *, \alpha)$ is a multiplicative left Homalternative algebra and essentially using (47) and (44). This finishes the proof.
Example 24. Let $A$ be a five-dimensional vector space with basis $\{e, u, v, w, z\}$ and let $\alpha: A \rightarrow A$ be a linear map given by

$$
\begin{aligned}
& \alpha(e)=e+u+v ; \\
& \alpha(u)=-u ; \\
& \alpha(v)=-v ; \\
& \alpha(w)=-w ; \\
& \alpha(z)=-z .
\end{aligned}
$$

Define on $A$ a binary operation “*” by

$$
\begin{aligned}
& e * e=e+u+v ; \\
& e * u=-v \\
& e * w=-w+z ; \\
& e * z=-z ; \\
& u * e=-u \\
& z * e=-z
\end{aligned}
$$

(again, only nonzero products are specified). Then $(A, *, \alpha)$ is a multiplicative right Hom-alternative algebra (see [23], Example 2.9). Then, using $[x, y]=x * y-y * x$ and (46), one could find (although the computation is somewhat lengthy) all the nonzero products "[, ]" and "(, , )" with respect to the basis elements $e, u, v, w$, and $z$ of $A$. We just point out that they are nonzero products; for example, $[e, u]=u-v,[e, w]=$ $-w+z,(e, u, e)=-u-v$, and $(e, w, e)=-w-z$. Therefore, Theorem 23 implies that $(A,[],,(,),, \alpha)$ is a Hom-Bol algebra.

\section{The Construction of Bol Algebras from Right Alternative Algebras Revisited}

As already mentioned in Section 2, for $\alpha=$ id in Definition 12 we get the definition of a left Bol algebra.

Definition 25 (see [25, 27]). A left Bol algebra is a triple $(A,[],,(,)$,$) in which A$ is a vector space, "[, ]" a binary operation, and " $(,,$,$) " a ternary operation on A$ such that

(B1) $[x, y]=-[y, x]$,

(B2) $(x, y, z)=-(y, x, z)$,

(B3) $\sigma_{x, y, z}(x, y, z)=0$,

(B4) $(x, y,[u, v])=[(x, y, u), v]+[u,(x, y, v)]+$ $(u, v,[x, y])-[[u, v],[x, y]]$

(B5) $(x, y,(u, v, w))=((x, y, u), v, w)+(u,(x, y, v), w)+$ $(u, v,(x, y, w))$,

for all $u, v, w, x, y, z \in A$

In this section we show how the construction of Hom-Bol algebras from right or left Hom-alternative algebras described in Section 4 can be specified to the ordinary untwisted case of construction of (left) Bol algebras from right or left alternative algebras $([29,31])$. In fact, for $\alpha=$ id in Theorem 23 and specifying the right alternative case, we get the following. 
Theorem 26. Let $(A, *)$ be a right alternative algebra. If one defines on A a ternary operation "(, , )" by

$$
(x, y, z)=[[x, y], z]-2 a s(z, x, y),
$$

where as $(u, v, w)=u v * w-u * v w$, then $(A,(,)$,$) is a Lts and$ $(A,[],,(,)$,$) is a left Bol algebra.$

Proof. Identities (B1) and (B2) are obvious. For $\alpha=\mathrm{id}$, the Hom-Jordan associator (see Definition 3 ) reduces to the usual Jordan associator $\operatorname{as}^{J}(u, v, w):=(u \circ v) \circ w-u \circ(v \circ w)$ in $(A, *)$. The fact that (B3) and (B5) hold in $(A, *)$ follows from the equality $(x, y, z)=\operatorname{as}^{J}(y, z, x)$ that holds in right alternative algebras (the untwisted form of (46)) and from that right alternative algebras are Jordan admissible [36]. Therefore $(A,(,)$,$) is a Lts since any Jordan algebra is a Lts$ with respect to the operation $(x, y, z)=\operatorname{as}^{J}(y, z, x)$ (see [1]). So we get the untwisted version of (HB5) and (HB7).

In order to show that $(\mathrm{B} 4)$ holds in $(A, *)$, we proceed as follows. First, recall that the identity

$$
\begin{aligned}
\text { as }(u v, y, x)= & u \text { as }(v, y, x)+\operatorname{as}(u, v, y) x \\
& +\operatorname{as}(u, v y, x)-\operatorname{as}(u, v, y x)
\end{aligned}
$$

holds in any algebra. Also, in a right alternative algebra $(A, *)$ (over a ground field of characteristic different from 2), the following identity holds [37]:

$$
\text { as }(u, v, v * y)=\text { as }(u, v, y) * v \text {; }
$$

that is, by linearization and right alternativity,

$$
\begin{aligned}
\text { as }(u, v * y, x)= & \text { as }(u, v, x * y)-\operatorname{as}(u, v, y) * x \\
& -\operatorname{as}(u, x, y) * v .
\end{aligned}
$$

Putting (60) in (58), we get

$$
\begin{aligned}
\text { as }(u * v, y, x)= & u * \text { as }(v, y, x)-\text { as }(u, x, y) * v \\
& + \text { as }(u, v,[x, y])
\end{aligned}
$$

that is, by right alternativity,

$$
\begin{aligned}
\text { as }(u * v, x, y)= & \text { as }(u, x, y) * v+u * \text { as }(v, x, y) \\
& -\operatorname{as}(u, v,[x, y]) .
\end{aligned}
$$

Now, in (62) switching $u$ and $v$ and then subtracting the obtained equality from (62), one gets

$$
\begin{aligned}
\text { as }([u, v], x, y)= & {[\operatorname{as}(u, x, y), v]+[u, \text { as }(v, x, y)] } \\
& +\operatorname{as}(v, u,[x, y])-\operatorname{as}(u, v,[x, y])
\end{aligned}
$$

(observe that (63) is the untwisted form of (41)). Next, write (57) as

$$
-2 \operatorname{ars}(z, x, y)=(x, y, z)-[[x, y], z] .
$$

Then multiplying (63) by -2 and using the equality above, and next proceeding as in the proof of Theorem 23 , one proves the validity of (B4) for $(A,[],,(,)$,$) . Thus we get that (A,[],,(,)$, is a left Bol algebra.
Remark 27. (i) The process of constructing left Bol algebras from right alternative algebras described in Theorem 26 above is different from the ones given in $[29,31]$. In our approach here, we rely essentially on fundamental properties of right alternative algebras (see, e.g., $[36,37]$ ) without subsidiary constructions.

(ii) If $(A, *)$ is a left alternative algebra, it is also possible to get a natural left Bol algebra structure on $(A, *)$. Indeed, one needs to consider the counterparts of $(x, y, z)$ and (63) that looks, respectively, as

$$
(x, y, z)=[[x, y], z]-\text { as }(x, y, z)
$$

(the untwisted version of (47)) and

$$
\begin{aligned}
\operatorname{as}(x, y,[u, v])= & {[\operatorname{as}(x, y, u), v]+[u, \text { as }(x, y, v)] } \\
& +\operatorname{as}([x, y], v, u) \\
& -\operatorname{as}([x, y], u, v) .
\end{aligned}
$$

Next one proceeds as in Theorem 26 observing that a left alternative algebra is also Jordan-admissible (see [36], Theorem 2, for right alternative algebras).

\section{Conflicts of Interest}

The authors declare that they have no conflicts of interests.

\section{References}

[1] N. Jacobson, "Lie and Jordan triple systems," American Journal of Mathematics, vol. 71, pp. 149-170, 1949.

[2] W. G. Lister, "A structure theory of Lie triple systems," Transactions of the American Mathematical Society, vol. 72, pp. 217-242, 1952.

[3] K. Yamaguti, "On algebras of totally geodesic spaces (Lie triple systems)," Journal of Science of the Hiroshima University, Series A, vol. 21, pp. 107-113, 1957/1958.

[4] S. Kobayashi and K. Nomizu, Foundations of Differential Geometry, Interscience Publishers, New York, NY, USA, 1963.

[5] O. Loos, Symmetric Spaces, vol. 1-2, W. A. Benjamin, New York, NY, USA, 1969.

[6] O. Loos, "Über eine beziehung zwischen malcev-algebren und lie-tripelsystemen," Pacific Journal of Mathematics, vol. 18, pp. 553-562, 1966.

[7] A. I. Maltsev, "Analytic loops," Matematicheskii Sbornik, vol. 78, pp. 569-578, 1955.

[8] A. A. Sagle, "Malcev algebras," Transactions of the American Mathematical Society, vol. 101, pp. 426-458, 1961.

[9] J. T. Hartwig, D. Larsson, and S. D. Silvestrov, "Deformations of Lie algebras using $\sigma$-derivations," Journal of Algebra, vol. 295, no. 2, pp. 314-361, 2006.

[10] A. Makhlouf and S. D. Silvestrov, "Hom-algebra structures," Journal of Generalized Lie Theory and Applications, vol. 2, no. 2, pp. 51-64, 2008.

[11] H. Ataguema, A. Makhlouf, and S. Silvestrov, "Generalization of n-ary Nambu algebras and beyond," Journal of Mathematical Physics, vol. 50, no. 8, Article ID 083501, 083501, 15 pages, 2009.

[12] S. Attan and A. N. Issa, "Hom-Bol algebras," Quasigroups and Related Systems, vol. 21, no. 2, pp. 131-146, 2013. 
[13] Y. Frégier, A. Gohr, and S. D. Silvestrov, "Unital algebras of Hom-associative type and surjective or injective twistings," Journal of Generalized Lie Theory and Applications, vol. 3, no. 4, pp. 285-295, 2009.

[14] D. Gaparayi and A. N. Issa, "A twisted generalization of LieYamaguti algebras," International Journal of Algebra, vol. 6, no. 5-8, pp. 339-352, 2012.

[15] A. Gohr, "On hom-algebras with surjective twisting," Journal of Algebra, vol. 324, no. 7, pp. 1483-1491, 2010.

[16] A. N. Issa, "Hom-Akivis algebras," Commentationes Mathematicae, vol. 52, no. 4, pp. 485-500, 2011.

[17] A. Makhlouf, "Paradigm of nonassociative Hom-algebras and Hom-superalgebras," in Proceedings of the Jordan Structures in Algebra and Analysis Meeting, pp. 143-177, Editorial Círculo Rojo, Almería, Spain, 2010.

[18] A. Makhlouf, "Hom-alternative algebras and Hom-Jordan algebras," International Electronic Journal of Algebra, vol. 8, pp. 177190, 2010.

[19] A. Makhlouf and S. Silvestrov, "Hom-algebras and Homcoalgebras," Journal of Algebra and Its Applications, vol. 9, no. 4, pp. 553-589, 2010.

[20] D. Yau, "Hom-Maltsev, Hom-alternative, and Hom-Jordan algebras," International Electronic Journal of Algebra, vol. 11, pp. 177-217, 2012.

[21] D. Yau, "On n-ary Hom-Nambu and Hom-Nambu-Lie algebras," Journal of Geometry and Physics, vol. 62, no. 2, pp. 506522, 2012.

[22] D. Yau, On n-ary Hom-Nambu and Hom-Maltsev Algebras, Cornell University, 2010.

[23] D. Yau, Right Hom-alternative algebras, Cornell University, New York, NY, USA, 2010.

[24] D. Yau, "Hom-algebras and homology," Journal of Lie Theory, vol. 19, no. 2, pp. 409-421, 2009.

[25] P. O. Mikheev, Geometry of smooth Bol loops [PhD Thesis], Friendship University, Moscow, Russia, 1986 (Russian).

[26] L. V. Sabinin and P. O. Mikheev, "Analytic Bol loops," in Webs and quasigroups, pp. 102-109, Kalinin Gos. University, Kalinin, Russia, 1982.

[27] L. V. Sabinin and P. O. Mikheev, "The geometry of smooth Bol loops," in Webs and Quasigroups, pp. 144-154, Kalinin Gos. University, Kalinin, Russia, 1984.

[28] T. B. Bouetou, "On Bol algebras," in Webs and Quasigroups, pp. 75-83, Tver State University, Tver, Russia, 1995.

[29] I. R. Hentzel and L. A. Peresi, "Special identities for Bol algebras," Linear Algebra and its Applications, vol. 436, no. 7, pp. 2315-2330, 2012.

[30] J. M. Pérez-Izquierdo, “An envelope for Bol algebras," Journal of Algebra, vol. 284, no. 2, pp. 480-493, 2005.

[31] P. O. Mikheev, "Commutator algebras of right-alternative algebras," Matematicheskii Issledovania, vol. 113, pp. 62-65, 1990.

[32] D. Yau, "Enveloping algebras of Hom-Lie algebras," Journal of Generalized Lie Theory and Applications, vol. 2, no. 2, pp. 95108, 2008.

[33] Y. Sheng, "Representations of hom-Lie algebras," Algebras and Representation Theory, vol. 15, no. 6, pp. 1081-1098, 2012.

[34] A. N. Issa, "On identities in Hom-Malcev algebras," International Electronic Journal of Algebra, vol. 17, pp. 1-10, 2015.

[35] Y. Nambu, "Generalized Hamiltonian dynamics," Physical Review D: Particles, Fields, Gravitation and Cosmology, vol. 7, pp. 2405-2412, 1973.
[36] A. A. Albert, "On the right alternative algebras," Annals of Mathematics: Second Series, vol. 50, pp. 318-328, 1949.

[37] E. Kleinfeld, "Right alternative rings," Proceedings of the American Mathematical Society, vol. 4, pp. 939-944, 1953. 


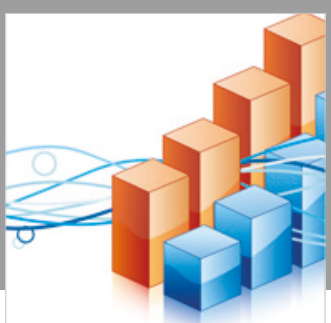

Advances in

Operations Research

\section{-n-m}
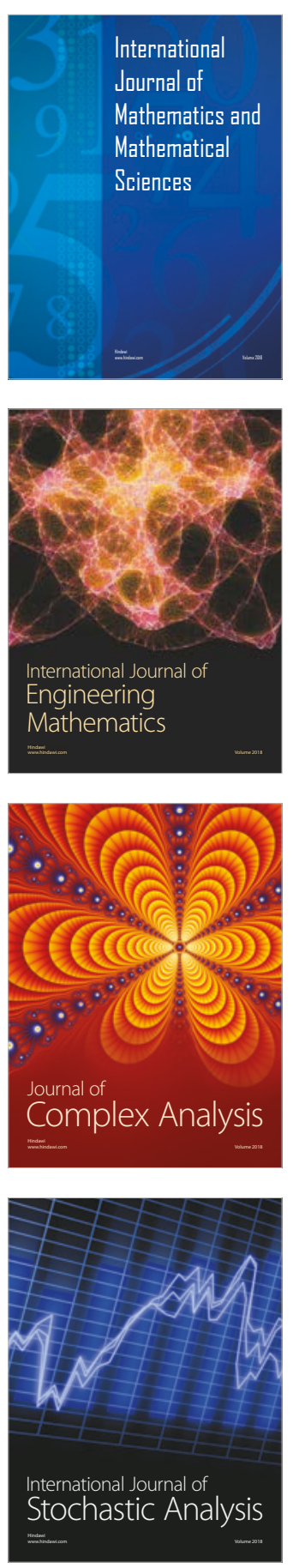
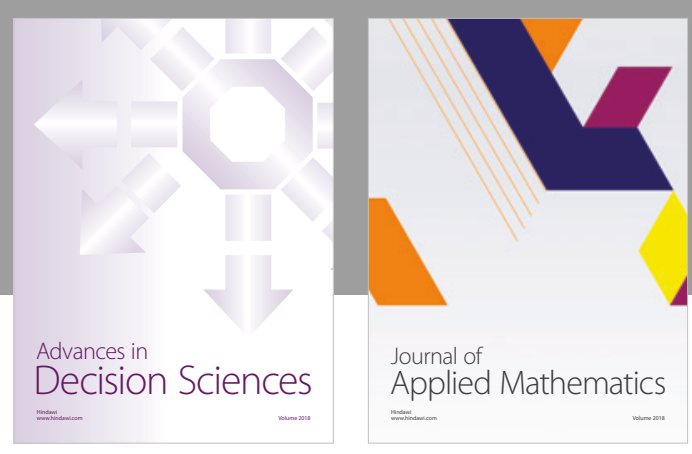

Journal of

Applied Mathematics
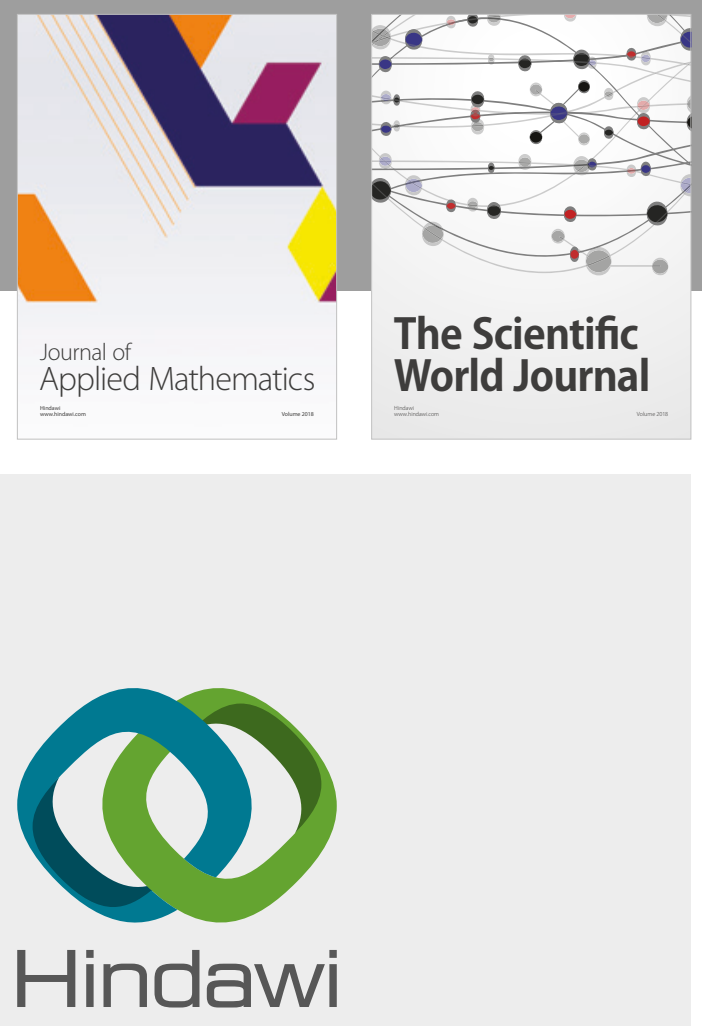

Submit your manuscripts at

www.hindawi.com

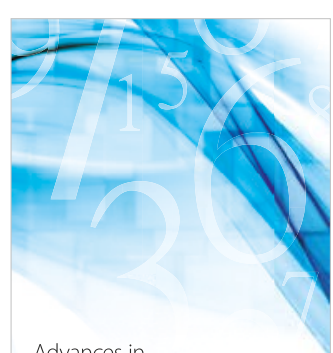

Advances in
Numerical Analysis
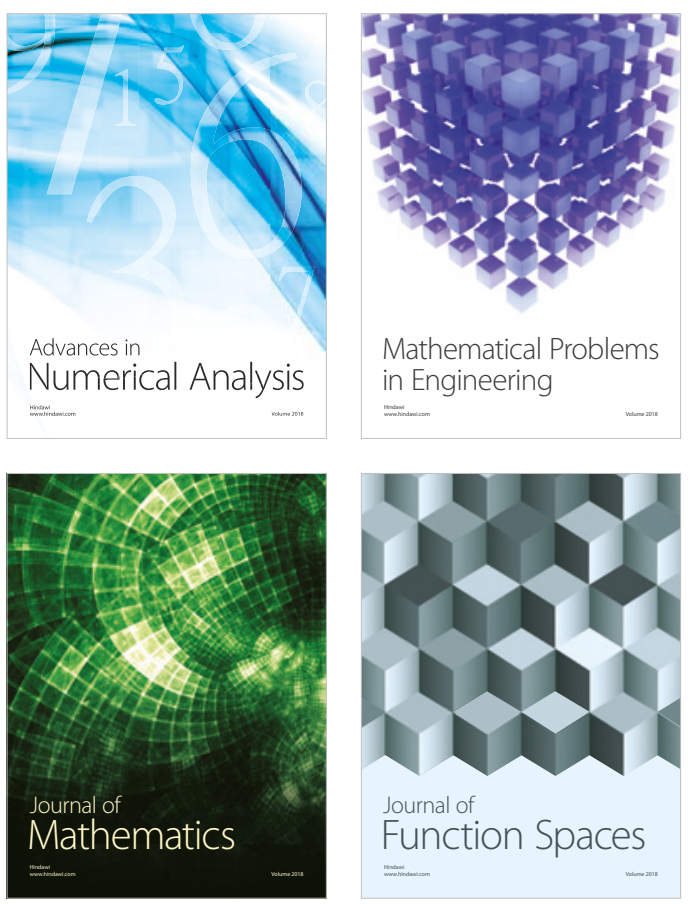

Mathematical Problems in Engineering

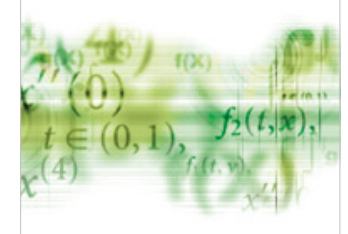

International Journal of

Differential Equations

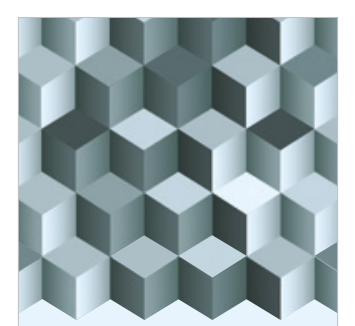

Journal of

Function Spaces

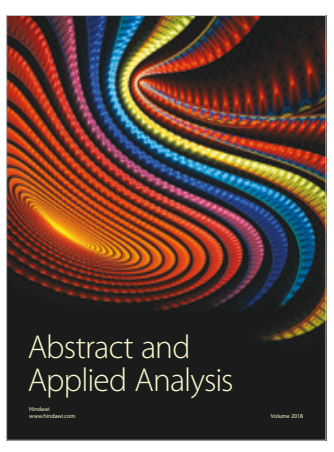

The Scientific

World Journal

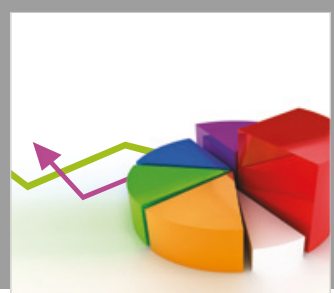

Journal of

Probability and Statistics
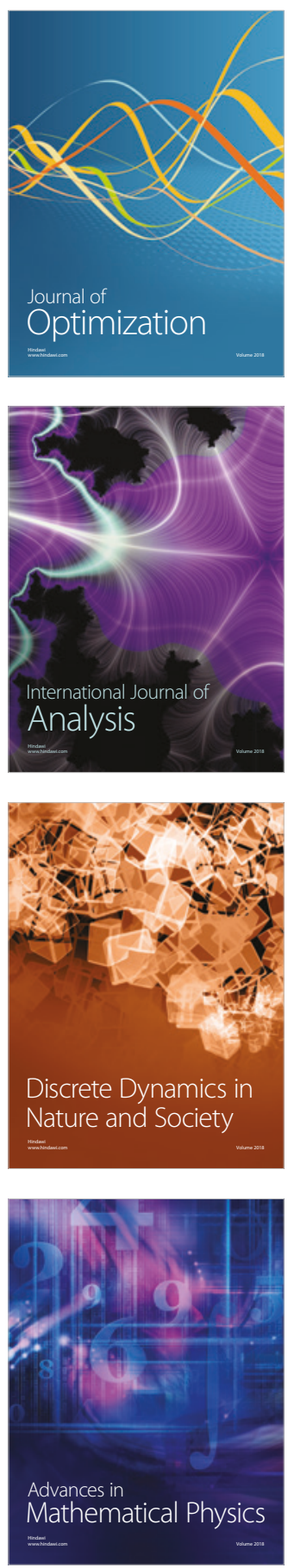